Received: April 03, 2017

Accepted: April 13, 2017

Published: May 02, 2017

\section{Laparoscopic Removal of a Migrated Intra Uterine De- vice Embedded in the Anterior Abdominal Wall in Yaounde (Cameroon), a Third World Country}

\author{
Bang Guy Aristide ${ }^{1 *}$, Nana Oumarou Blondel ${ }^{1}$, Nong Libend Gilles Thierry ${ }^{2}$, Noah Noah \\ Dominique $^{3}$, Savom Eric Patrick ${ }^{1}$ \\ ${ }^{1}$ Visceral and laparoscopic surgery unit, National Social Insurance Fund Health Center of \\ Yaoundé CAMEROON. \\ ${ }^{2}$ Gynecologic and obstetric unit, National Social Insurance Fund Health Center of \\ Yaoundé CAMEROON \\ ${ }^{3}$ Department of Gastroenterology, Central Hospital of Yaoundé CAMEROON.
}

*Corresponding author: Bang Guy Aristide, National Social Insurance Fund Health Center of Yaoundé, B.P: 5777, Yaoundé Cameroon, Tel:+237699125324;E-mail: guyaristidebang@yahoo.fr

\section{Abstract}

\subsection{Background}

Uterine perforation is a serious complication which can happen after intrauterine device (IUD) insertion. Following the uterine rupture, an IUD may migrate into gynecologic, urinary or gastro-intestinal system organs. There are many reports of migrated iuds but fewer report of iuds embedded in the abdominal wall. Laparoscopic removal of a migrated IUD wasn't yet described in our country.

\subsection{Case Presentation}

We report a case of a 32-year-old Cameroonian woman who presented to our gynecologic unit for a follow-up visit 3 months after uncomplicated IUD insertion. The IUD' string wasn't found. Abdominal CT-scan showed the IUD embedded in the anterior abdominal wall. Through a laparoscopic approach, the device was removed.

\subsection{Conclusion}

Surgical removal of a migrated IUD reduces the possible risks of abdominal complications. The laparoscopic approach for migrated IUD removal may be simple and safe even in developing countries as our own.

\section{Keywords}

Intrauterine device migration ; Cameroon ; Laparoscopy ; Uterine perforation

\section{Introduction}

Intrauterine device (IUD) introduce in 1909 by RICHTER, is worldwide the most popular form of reversible contraception [1], with an estimated 175 million women using it in 2007 [2].

Complications of IUD insertion are uncommon but may include expulsion, retraction into the cervix or uterus, bleeding, infection, ectopic pregnancy and perforation through the uterine wall and into the abdominal cavity or neighboring organs. Among these, perforation is the most serious, with an incidence reported to be between 1.3 and 1.6 per 1000 insertions [3]. Perforations tends to occur in the immediate post- insertion period, especially during the first 6 months [4], but cases of uterine perforation have also been noted several years after insertion [5,6]. After perforation, the device can migrate into the gynecologic, urinary or gastrointestinal system organs. We report the first case of a laparoscopic removal of a migrated IUD in Cameroon.

\section{Case Presentation}

A 32-year-old woman, gravida 3, para 3, was presented to our gynecology unit for a follow-up visit 3 month after uncomplicated IUD insertion. One month before, she began complaining of intermittent hypogastric pain. On vaginal examination, the string wasn't found. An abdominal X-ray depicted the IUD in the abdominal cavity at hypogastric region (Figure 1). A computerized tomography of the pelvis and abdomen confirmed the IUD in the abdominal cavity, embedded in the hypogastric wall (Figure 2).

The patient was then transferred to the visceral and laparoscopic unit for removal. Upon admission, she underwent a physical examination that detected no abnormalities, apart from light tenderness upon hypogastric palpation. Preoperative workshops were normal. A laparoscopic approach was decided. An urinary tube was inserted. 


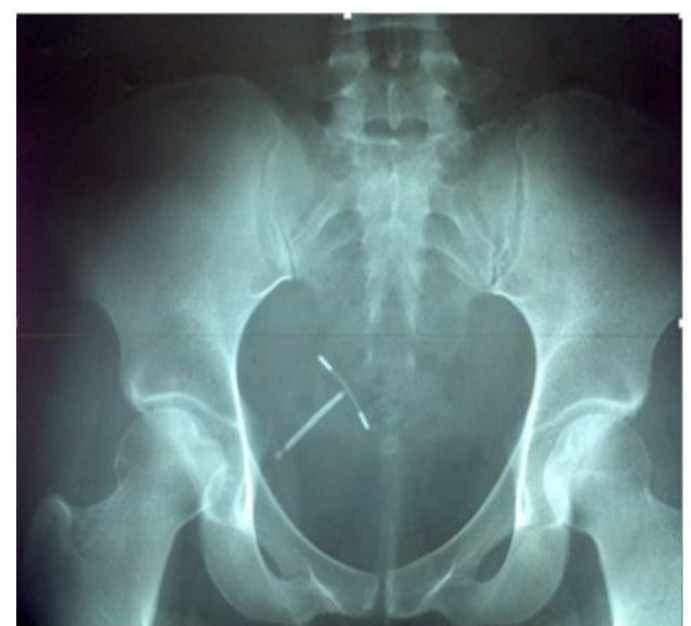

Figure 1: Abdominal X-ray

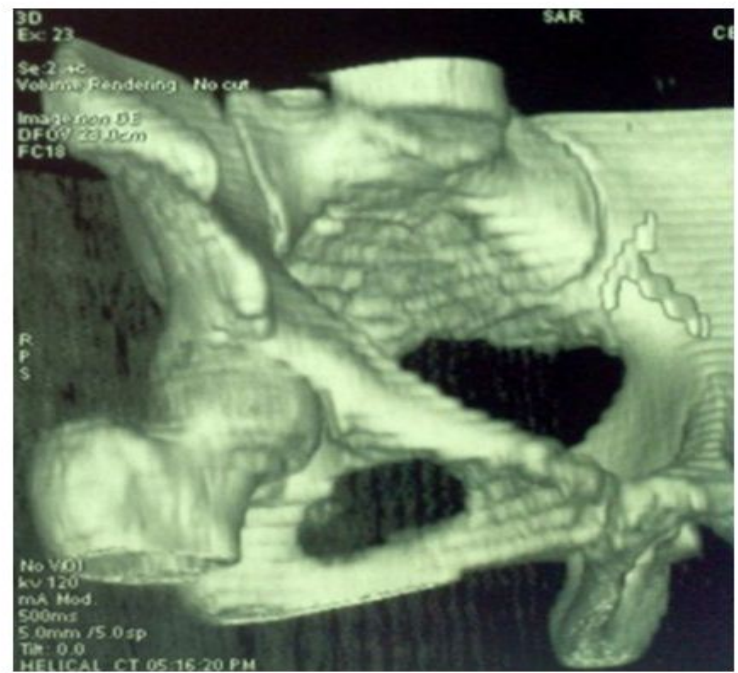

Figure 2: Abdominal CT-scan showing IUD embedded in the anterior abdominal wall.

We introduce the first $10 \mathrm{~mm}$ optic access supraumbilical by "open-coelioscopy". The IUD was found embedded into the hypogastric wall, omentum attached on it, with the IUD string in the peritoneal cavity (Figure 3). Uterus and adnexa were normal. Two working $5 \mathrm{~mm}$ trocars were then inserted one in each flank. The omentum was disinserted, the parietal peritoneum excised and the device removed (Figure 4). The post-operative course was uneventful with discharge at day 1 . The cosmetic result was good (Figure 5).

\section{Discussion}

Must cases of migrated iuds are asymptomatic and fewer will present with acute symptoms [7]. This leaves close follow-up after IUD placement as the best course of action to preemptively identify IUD perforation as is highlighted in our case.

The management of intraperitoneal IUD in asymptomatic or pauci-symptomatic patients is somewhat controversial. World Health Organization guidelines recommend removal of migrating

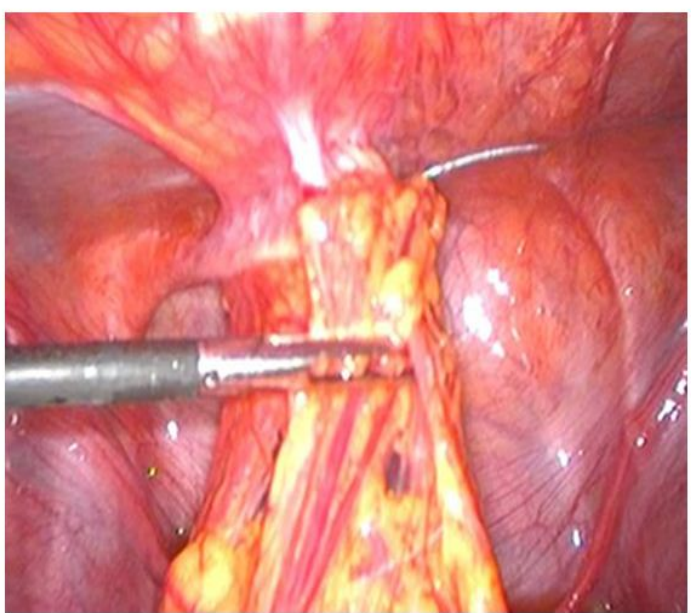

Figure 3: Laparoscopic view of migrating IUD embedded in the hypogastic anterior wall with omemtum attached on it and the string free in the peritoneal cavity.

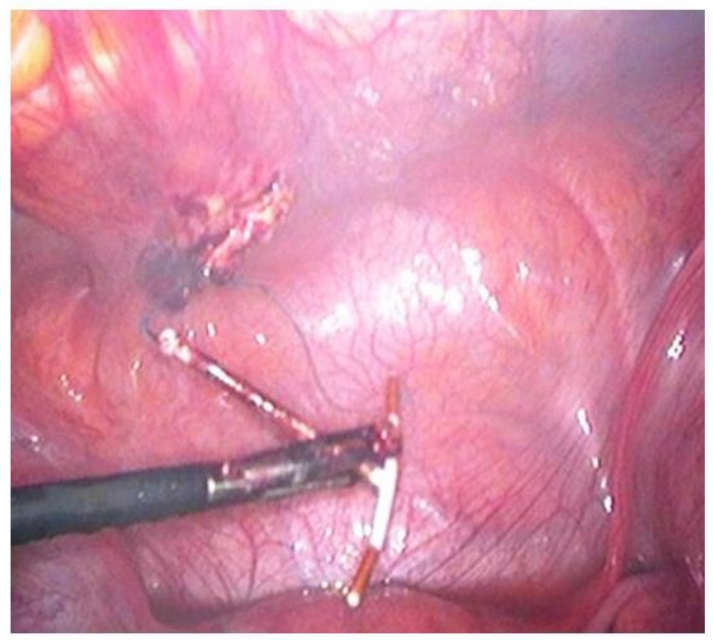

Figure 4: IUD removal.

IUD irrespective of their type and location [7]. It is this recommendation we followed in our case since our patient was paucisymptomatic.

In a recent literature review, MOSLEY et al [7] noted about 129 cases that perforated IUD have been found in many locations including free in pelvis(32,5\%), embedded in the omemtum $(31,7 \%)$, attached to bowel $(10 \%)$, attached to uterus $(6,9 \%)$, free in peritoneal cavity $(4,6 \%)$, attached to broad ligament $(3,8 \%)$, mass of bowel and pelvic structures $(3,1 \%)$, mass of omemtum and pelvic structures $(2,3 \%)$, attached to rectum $(2,3 \%)$, attached to tubo-ovarian $(1,5 \%)$ and attached to bladder $(1,3 \%)$. The location of the IUD in our case, embedded in the anterior abdominal wall, appear then to be particular and uncommon.

Laparoscopic removal of intra-abdominal IUD should be the preferred choice of surgical management $[7,8]$. Laparoscopy is a safe and minimally invasive procedure with less complications, 


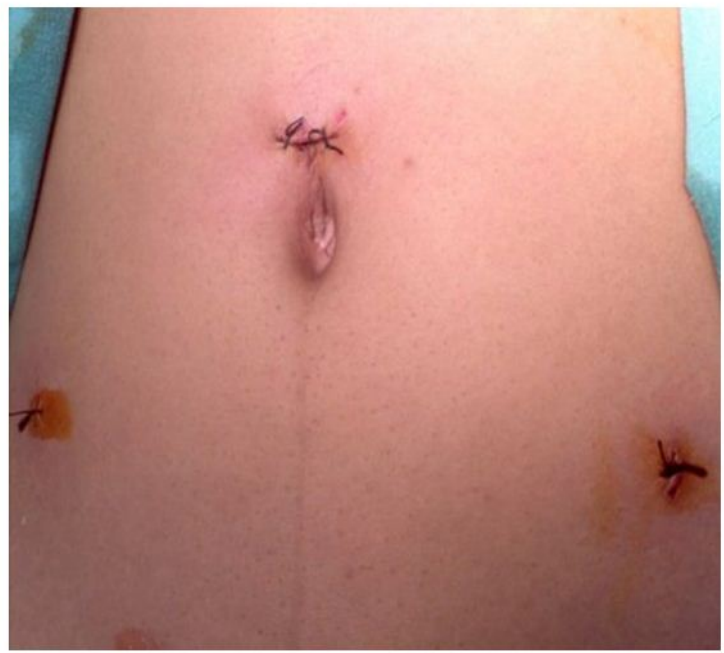

Figure 5: Cosmetic results.

shorter operative time and hospitalization compared to laparotomy. In our practice, this was the first time we encountered this clinical scenario. In our knowledge it is the first published case of IUD migration in our country. It can be explained by the fact that physicians perceive migrated IUD as result of malpractice and remove it without any report. We encourage surgeons to report these cases and use laparoscopic approach for removal.

\section{Conclusion}

Our patient presented with a particular location of a migrated IUD, embedded in the anterior abdominal wall. A migrated IUD should be removed when the diagnosis is made. Laparoscopy approach, as such in this case, can be performed for removal. We encourage this approach for migrated IUD removal even in developing countries as our own.

\section{Authors' Contribution}

All persons designated as authors above, qualify for authorship, they took active part in the management of these patients and write up of this manuscript. All authors have read and agreed to the final manuscript.

\section{References}

1. United Nations Department of Economic and Social Affairs Population Division. World Contraceptive use 2009.

2. Sivin I, Batar I. State-of-the-art of non-hormonal methods of contraception: III. Intrauterine devices. Eur J Contracept. 2010;15(2):96-112. Doi: 10.3109/13625180903519885

3. Arslan A, Kanat-Pektas M, Yesilyurt H, Bilge U. Colon penetration by a copper intrauterine device: a case report with literature review. Arch Gynecol Obstet . 2009;279(3):395-397. Doi: $10.1007 /$ s00404-008-0716-2

4. Heartwell SF, Schlesselman S. Risk of uterine perforation among users of intrauterine devices. Obstet Gynecol. 1983;61(1):31-36.

5. Aydogdu O, Pulat H. Asymptomatic far-migration of an intrauterine device into the abdominal cavity: A rare entity. CUAJ. 2012;6(3):E134-E136. Doi: 10.5489/cuaj.11100

6. Stuckey A, Dutreil P, Aspuru E, Nolan TE. Symptomatic cecal perforation by an intrauterine device with appendectomy removal. Obstet Gynecol. 2005;105(5,part2):1239-1241. DOI:10.1097/01.AOG.0000157760.59342.bc

7. Mosley FR, Shahi N, Kurer MA. Elective surgical removal of migrated intrauterine contraceptive devices from within the peritoneal cavity: A comparison between open and laparoscopic removal. JSLS. 2012;16(2):236-241.

8. Weerasekera A, Wijesinghe P, Nugaduwa N. Sigmoid colocolic fistula caused by intrauterine device migration: a case report. Journal of Medical Case Reports. 2014;8:81. 